\title{
Búsqueda activa y evaluación de factores de riesgo cardiovascular en adultos jóvenes, Cartagena de Indias, 2007
}

\author{
Alicia Norma Alayón, Saudith Ariza, Karen Baena, Lina Lambis, Lina Martínez, Lourdes Benítez \\ Instituto de Investigaciones Biomédicas Alberto Montealegre, IDIBAM, Facultad de Ciencias de la Salud, \\ Universidad de San Buenaventura, Seccional Cartagena, Cartagena, Colombia
}

Introducción. Algunos estudios de gran alcance sugieren que los individuos con alto riesgo cardiovascular en la mediana edad pueden ser identificados por una mayor presencia de factores de riesgo en edades tempranas.

Objetivos. Estimar la prevalencia y la agrupación de factores de riesgo biológicos y de comportamiento de enfermedad cardiovascular en adultos jóvenes en un barrio de Cartagena de Indias.

Materiales y métodos. Se llevó a cabo un estudio descriptivo de corte transversal en 207 personas de 20 a 44 años, con aplicación de entrevistas, mediciones antropométricas y tomas de muestras sanguíneas para evaluar la glucemia y el perfil lipídico. Resultados. El 80\% (IC95\% 73,6-86,4) presentó historia familiar de enfermedades cardiovasculares. Se halló una prevalencia de 37\% (IC95\% 30,4-43,6) para factores de riesgo conductuales, como la inactividad física, y de 66\% (IC95\% 59,5-72,5) para el consumo de dieta aterogénica. El consumo de alcohol y tabaco fue significativamente mayor en los varones. La prevalencia de factores de riesgo biológicos fue de 42\% (IC95\% 35,3-48,7) para cHDL (high-density lipid cholesterol) bajo, de 39\% (IC95\% 32,4-45,6) para hipertrigliceridemia, de 34\% (IC95\% 27,5-40,5) para CLDL (low density lipid cholesterol) elevado y de $21 \%$ (IC95\% 15,5-26,5) para síndrome metabólico. Se observó presencia de tres o más factores de riesgo biológico en el 30\% (IC95\% 23,8-36,2); de uno o dos, en el 54\% (IC95\% 47,2-60,8), y el 16\% (IC95 \% 11,0-21,0) de los participantes no presentó ninguno de ellos. Conclusión. La alta prevalencia de factores de riesgo cardiovascular en adultos jóvenes subraya la importancia de las políticas encaminadas a reducirlos mediante estrategias de prevención.

Palabras clave: enfermedades cardiovasculares, dislipidemias, síndrome X metabólico, factores de riesgo, estilo de vida, grasa intraabdominal, promoción de la salud.

Active search and assessment of cardiovascular risk factors in young adults, Cartagena de Indias, 2007

Introduction. Extensive clinical and statistical studies suggest that individuals with high overall cardiovascular risk in midlife can be identified by their relatively higher values of risk factors at younger ages.

Objectives. To estimate the prevalence and clustering of recognized biological and behavioral cardiovascular disease risk factors among young adults in a neighborhood in Cartagena de Indias.

Methods. A cross-sectional descriptive study was performed. A sample of 207 young adults were interviewed in order to obtain information regarding their anthropometric and behavioral characteristics. Each individual's standardized glucaemia and serum lipids were measured.

Results. In $80 \%$ (95\%Cl: 73.6-86.4) family history of cardiovascular disease was present; $37 \%(95 \% \mathrm{Cl}$ : 30.4-43.6) had behavioral risk factors including physical inactivity, and in $66 \%(95 \% \mathrm{Cl}: 59.5-72.5)$ atherogenic diet. Alcohol and tobacco consumption were significantly higher in males. Biological risk factor prevalence was 42\% (35.3-48.7) for low cHDL, 39\% (IC95\%: 32.4-45.6) for hypertriglyceridemia, 34\% (IC95\%:27.5-40.5), high cLDL, and 21\% (IC95\%: 15.5-26.5) for metabolic syndrome. The presence of three or more biological risk factors occurred in 30\% (IC95\%: 23.8-36.2); one or two in 54\% (IC95\%: 47.2-60.8), and 16\% (IC95\%: 11.0-21.0) of the participants presented no risk factors.

Conclusion. The high prevalence of cardiovascular risk factors in young adults underscores the importance of policies aimed at reducing these factors by prevention strategies.

Key words: cardiovascular diseases, dyslipidemias, metabolic syndrome X, risk factors, life style, intra-abdominal fat, health promotion. 
En la actualidad, las enfermedades crónicas no trasmisibles representan la principal causa de muerte en la población mundial, principalmente debido a los cambios nutricionales y al proceso de urbanización creciente de los últimos tiempos (1).

Se estima que las muertes por enfermedades cardiovasculares serán responsables de casi el $50 \%$ de las defunciones por enfermedades no transmisibles hasta el año 2020 , pero su proporción aumentará a $34 \%$ del total de muertes en el año 2010 y a $37 \%$ en el 2020 (2).

En conjunto, la enfermedad isquémica del corazón y la cerebrovascular producen algo más de $60 \%$ de la mortalidad cardiovascular total, y son las causas con mayor impacto en la población adulta $(3,4)$. Por tal razón, organizaciones, como la Organización Mundial de la Salud (OMS) y la Organización Panamericana de la Salud (OPS), han diseñado estrategias con el objetivo de mejorar la salud en las Américas, que buscan la reducción de los múltiples factores de riesgo para este tipo de enfermedades. Entre dichas estrategias, resalta el Conjunto de Acciones para la Reducción Multifactorial de las Enfermedades No Trasmisibles, conocido por sus siglas CARMEN $(5,6)$, que propone el estudio y la vigilancia de estas entidades en todos los niveles de organización de la población, empezando por la unidad básica, es decir los barrios, como una alternativa para la detección temprana y la intervención oportuna y holística de los factores de riesgo.

Éstos se agrupan en tres categorías: generales o no modificables, como la edad, el sexo y los genes; conductuales o modificables, como el tabaquismo, la dieta inadecuada, el alcoholismo y el sedentarismo; e intermedios, como dislipidemias, hiperglucemia, hipertensión arterial y obesidad (7). Todos ellos están fuertemente ligados a la enfermedad coronaria y a la aterogénesis, y muchos de ellos son condicionados por variables socioambientales (8) y de comportamientos adquiridos desde edades tempranas del desarrollo.

\section{Materiales y métodos}

Se llevó a cabo un estudio de enfoque cuantitativo, descriptivo, de corte transversal, en 207 adultos

Correspondencia:

Alicia Norma Alayón, Manga, Cuarto Callejón, Urbanización

Santa Cruz, casa № 3, Cartagena de Indias, Colombia

Teléfono: 660 5441, celular: (315) 7760526

alinorala@gmail.com

Recibido: 09/10/09; aceptado:18/02/10 jóvenes con edades entre 20 y 44 años, habitantes del barrio Ternera sector San Carlos, de la localidad tres, comuna trece de Cartagena de Indias, durante el último semestre del año 2008.

El tamaño de muestra representativa se determinó mediante una fórmula estadística para población finita, asumiendo un $5 \%$ de error y un nivel de confianza del $95 \%$ a partir de los datos arrojados por un censo de la población casa a casa, realizado por el mismo grupo de investigadores, que arrojó un total de 480 individuos en ese rango de edad.

La selección se realizó de manera aleatoria. A todos los sujetos se les aplicó un cuestionario de entrevista, adaptado a partir del método paso a paso recomendado por la OPS, para recolectar, analizar y diseminar información sobre factores de riesgo, el cual recibe el nombre de STEPS panamericano; su objetivo fue recabar la información necesaria consistente en datos sociodemográficos y antropométricos, hábitos alimentarios, actividad física, consumo de alcohol, tabaquismo y antecedentes familiares, previa lectura y firma de una cláusula de consentimiento informado elaborada acorde a los principios que rigen la investigación en humanos (Resolución 08430/1993).

Se consideraron como hábitos alimentarios inadecuados, o dieta aterogénica, al elevado consumo de grasas y azúcares refinados, al bajo consumo de frutas y verduras o ambos; el sedentarismo se asumió como la realización de menos de 30 minutos de ejercicio vigoroso, por lo menos, tres veces por semana. También, se determinó el consumo de alcohol en cantidad superior a 80 gramos diarios y el consumo de cigarrillos en el momento de la entrevista o en los tres meses inmediatamente anteriores, lo cual se consideró como tabaquismo.

Se tomó como hipertensión arterial una tensión arterial sistólica mayor de $130 \mathrm{~mm} \mathrm{Hg}$, una diastólica mayor de $85 \mathrm{~mm} \mathrm{Hg} \mathrm{o} \mathrm{ambas,} \mathrm{medidas}$ de acuerdo con las recomendaciones de la American Heart Association y la American Society of Hypertension.

Las muestras de sangre se obtuvieron por punción venosa y se transportaron de manera inmediata y refrigerada para la separación del suero por centrifugación a $3.500 \mathrm{rpm}$ durante 5 minutos en un tiempo que no superara los 30 minutos. Mediante técnicas espectrofotométricas, se determinaron el perfil lipídico y la glucemia 
en ayunas. El protocolo completo del estudio fue sometido a evaluación y aprobación por parte del Comité de Ética Institucional de la Universidad de San Buenaventura, Seccional Cartagena.

Para el análisis e interpretación de los datos, se utilizó el software estadístico Statgraphics Plus, versión 5 , asumiendo un nivel de significancia de 0,05 y una confianza del $95 \%$ para las decisiones.

\section{Resultados}

De las 207 personas incluidas en el estudio, 150 $(72 \%)$ correspondieron al sexo femenino y 57 (28\%) al masculino. La edad promedio fue de 33,5 años (IC95\% 32,3-34,7).

La distribución general de los factores de riesgo cardiovascular en la población de adultos jóvenes del sector estudiado, se presenta en la figura 1.

El $80 \%$ (IC95\% 73,6-86,4) de las personas refirieron tener, al menos, un familiar en primer grado de consanguinidad que hubiera padecido alguna de las enfermedades que la revisión de literatura menciona como relacionadas con el riesgo cardiovascular (9-12). La herencia familiar más frecuente fue la hipertensión arterial, 58\% (IC95\% $51,3-64,7)$, seguida por hipercolesterolemia, $43 \%$ (IC95\% 36,3-49,7), diabetes, 37\% (IC95\% 30,443,6 ) e infarto agudo temprano, 29\% (IC95\% 22,8$35,2)$.

El estudio de los estilos de vida y de las medidas antropométricas mostró, en su orden, que los hábitos alimenticios inadecuados y la adiposidad intraabdominal comparten el primer lugar de prevalencia en los adultos jóvenes en $66 \%$ de los casos (IC95\% 59,5-72,5). El 37\% (IC95\% 30,4-
43,6) reportó actividad física menor de 30 minutos tres veces por semana, lo cual se consideró como indicador de sedentarismo. El consumo de alcohol y el tabaquismo se presentaron en 11\% (IC95\% $6,7-15,3)$ de los entrevistados, mientras que 9\% (IC95\% 5,1-12,9) presentaba presión arterial superior a 130/85 mm Hg, sistólica y diastólica respectivamente.

El análisis estadístico no arrojó diferencia significativa al comparar el comportamiento entre sexos para la mayoría de los factores de riesgo mostrados en la figura anterior, con la excepción del hábito de fumar, que presentó mayor frecuencia en hombres que en mujeres y cuyos resultados se muestran en la figura 2 (IC95\% 10-32 en hombres; IC95\% 3-11 en mujeres; $p<0,001$ ), situación similar al comportamiento del consumo de alcohol (IC95\% 19-43 en hombres; IC95\% 0,3-6 en mujeres; $p<0,001)$.

De los hábitos indagados (tabaquismo, sedentarismo, dieta inadecuada y consumo de alcohol), 47\% (IC95\% 40,2-53,8) del total de la población estudiada presentaba simultáneamente dos hábitos inadecuados, y la combinación más frecuente fue la de hábitos alimenticios y sedentarismo. El 32\% (IC95\% 25,6-38,4) de los entrevistados presentó tres o más de estos factores en el momento del estudio.

La figura 3 muestra la distribución porcentual de la alteración de las variables bioquímicas respecto de los niveles recomendados; la más frecuente fue la disminución de colesterol en lipoproteínas de alta densidad [cHDL (high-density lipid cholestero), menor de $40 \mathrm{mg} / \mathrm{dl}$ ], el incremento del colesterol total

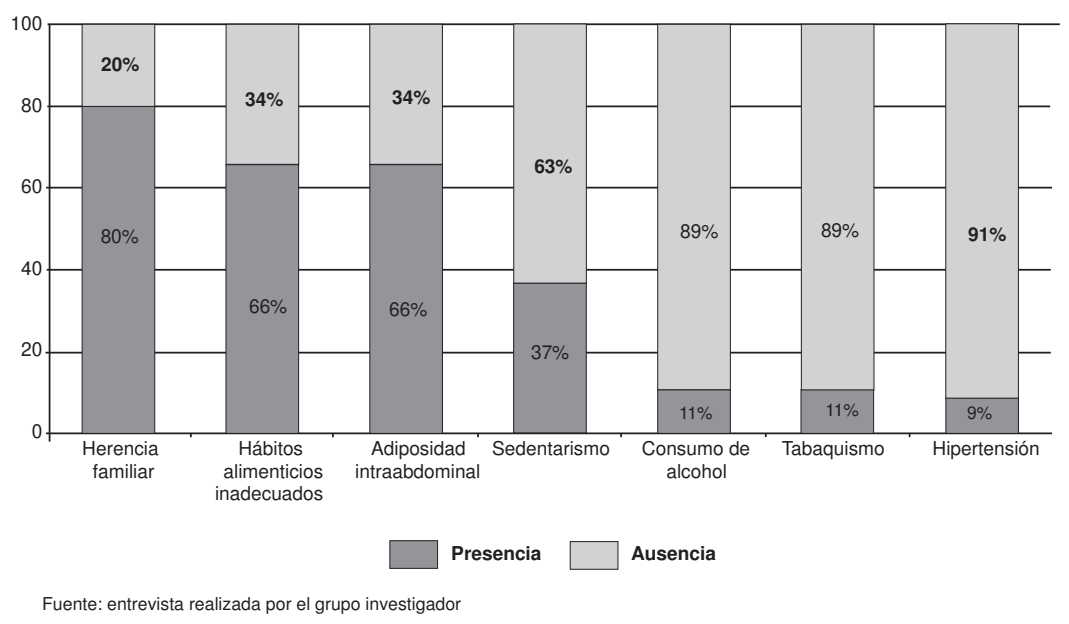

Figura 1. Distribución porcentual de la presencia de factores de riesgo cardiovascular 


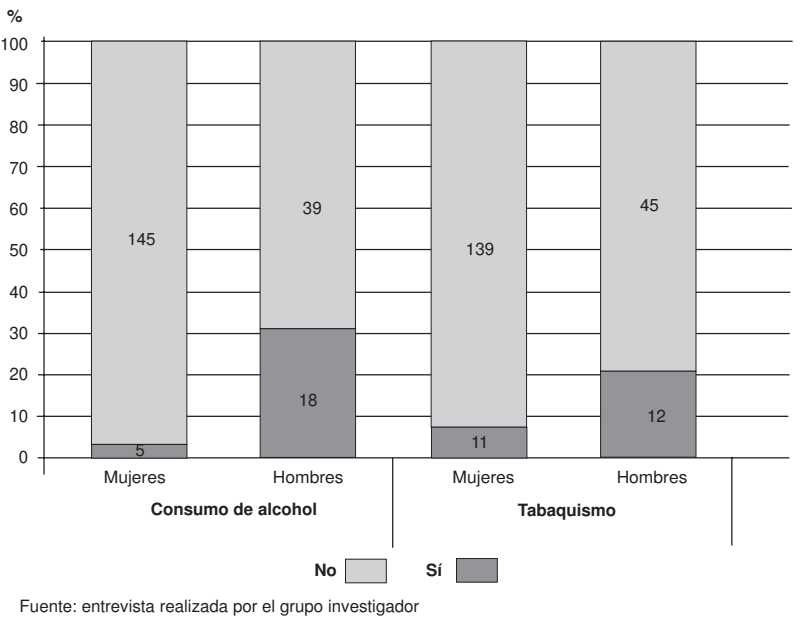

Figura 2. Distribución porcentual comparativa de riesgo cardiovascular entre hombres y mujeres, debido a consumo de alcohol y tabaquismo.

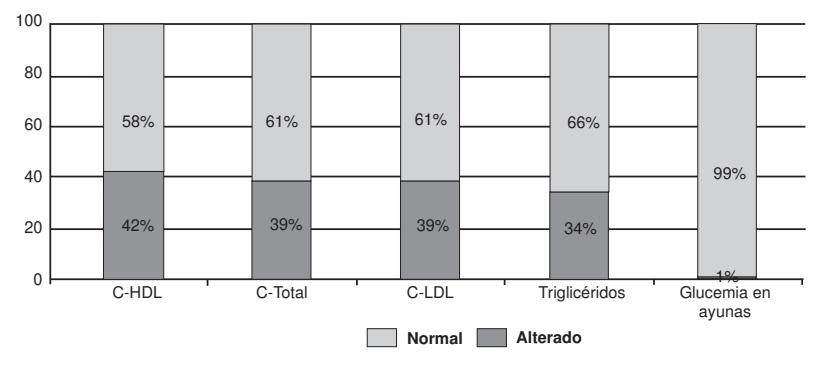

Fuente: determinaciones bioquímicas de laboratorio realizadas por los investigadores

Figura 3. Distribución porcentual de la presencia de alteración en las variables bioquímicas estudiadas

(mayor de $200 \mathrm{mg} / \mathrm{dl}$ ) y colesterol de lipoproteínas de baja densidad [CLDL (low-density lipid cholesterol), mayor de $130 \mathrm{mg} / \mathrm{dll}$.

El 16\% (IC95\% 11,0-21,0) de las personas incluidas en el estudio no presentaban ninguno de los siguientes factores de riesgo: hipertensión arterial, adiposidad intraabdominal, $\mathrm{cHDL}$ disminuido $\mathrm{O}$ aumento de cLDL, glucemia o de triglicéridos, mientras que el 54\% (IC95\% 47,2-60,8) presentó uno o dos de ellos de forma simultánea y el 30\% (IC95\% 23,8-36,2) restante, más de tres.

Utilizando los criterios de la International Diabetes Federation (2005), el 21\% (IC95\% 15,5-26,5) de la población incluida en este estudio presentaba síndrome metabólico, al reunir un perímetro de cintura mayor de $90 \mathrm{~cm}$ en hombres o de $80 \mathrm{~cm}$ en mujeres, con dos o más de los siguientes criterios menores: tensión arterial mayor de 130/85 mm Hg), triglicéridos superiores a $150 \mathrm{mg} / \mathrm{dl}$, cHDL menor de $40 \mathrm{mg} / \mathrm{dl}$ en hombres o $50 \mathrm{mg} / \mathrm{dl}$ en mujeres, y glucemia en ayunas mayor de $100 \mathrm{mg} / \mathrm{dl}$ o diabetes preexistente (13-15).

\section{Discusión}

Para la selección de las personas participantes del estudio no se tuvo como criterio específico de inclusión el sexo, verificándose que el mayor porcentaje de los participantes fueron mujeres, lo que posiblemente se debe a que este grupo de población cuenta con mayor disponibilidad de tiempo en los horarios en que se realizaron los muestreos, debido a la necesidad del estado de ayuno para las pruebas de laboratorio. Es importante considerar este factor limitante en el momento de analizar los resultados globales que pudieran estar influenciados por el predominio de la población femenina en el estudio.

La herencia familiar, como factor de riesgo inmodificable, muestra que las condiciones como hipertensión arterial, hipercolesterolemia, diabetes e infarto cardiaco temprano (16), derrame cerebral y cáncer, se encuentran registradas como antecedentes en la mayor parte del grupo estudiado; esto coincide con lo hallado en estudios previos en estudiantes universitarios, en los que se obtuvo que un porcentaje igual a $80,5 \%$ de los encuestados afirmaron tener antecedentes familiares de diabetes, obesidad, hipertensión, enfermedades cardiovasculares o hipercolesterolemia, en el mismo orden de distribución (17).

Entre los factores de riesgo conductuales, los que muestran mayor presencia en la población adulta joven del sector son los hábitos alimentarios inadecuados acompañados de adiposidad intraabdominal, resultados con comportamiento similar al obtenido en estudios realizados en México y Chile $(18,19)$ y que coinciden con las tendencias mundiales en estos últimos años de incremento de masa grasa, obesidad y sobrepeso.

En tal sentido, tanto hombres como mujeres tienen dietas con alto consumo de grasas saturadas, ácidos grasos trans, colesterol y carbohidratos, hábitos que contribuyen en gran parte a la formación y acumulación de grasa abdominal, y que se han sido asociado como un factor de riesgo directo para la aparición de enfermedades cardiovasculares (18-20) y, en forma similar a lo hallado en otros estudios, escaso consumo de frutas y verduras, que se consideran factores protectores (21).

Por su parte, al comparar personas con obesidad intraabdominal y sin ella, el estudio no mostró una relación estadísticamente significativa con respecto al sedentarismo, altamente prevalente en todos los grupos, lo cual podría deberse al tamaño o a la 
composición de la muestra incluida en el estudio o estar relacionado con tiempos insuficientes de ejercicio o guardar relación con lo afirmado por otros autores quienes aseguran que "el ejercicio parece desempeñar un modesto papel en la pérdida de peso" (22), aun cuando los beneficios metabólicos derivados de éste se han demostrado sólidamente y sitúan a este hábito como una de las principales dianas de los programas educacionales para implementar con este grupo de población en el futuro.

En lo que respecta al tabaquismo y al consumo de alcohol, su frecuencia es similar entre sí y mayor en el grupo de los hombres, comparado con el de las mujeres, con perfiles similares a lo hallado por otros autores (23). Esto puede explicarse por la influencia que genera el entorno cultural, lo cual coincide con lo expresado en la Encuesta Nacional de Adicciones 2002 cuando se afirma que "desde la adolescencia, el consumo de alcohol empieza a ser mucho más frecuente en hombres que en mujeres" (24), y en jóvenes, el incremento de riesgo por su consumo es significativamente mayor que en adultos (25), aun cuando las evidencias actuales todavía son controversiales (26).

Muy relacionado con lo anterior se presenta el comportamiento de las variables bioquímicas en la población de estudio, en especial, aquéllas que se relacionan con el perfil de lípidos y que se constituyen en un factor de riesgo alto para enfermedad cardiovascular (27).

En este sentido, la alteración más frecuente correspondió a cifras de cHDL inferiores a los rangos recomendados, comportamiento análogo al observado en investigaciones realizadas de manera previa por otros investigadores (28). Estos datos, así como el elevado porcentaje de personas que presentaron valores séricos de colesterol total, cLDL y triglicéridos por encima de los de referencia, corresponden a lo esperado, habida cuenta del alto porcentaje de la población que consume una alimentación rica en grasas y carbohidratos; este conjunto representa un aumento significativo en el riesgo de desarrollar procesos aterogénicos en el futuro.

La glucemia en ayunas elevada permitió el diagnóstico de diabetes en dos de la tres personas que la presentaron y que lo ignoraban hasta el momento del presente estudio, lo que confirma que, en países en desarrollo como Colombia, aún son muchos los diabéticos que desconocen su situación (29).
Los resultados obtenidos, incluyendo la presencia simultánea de dos o más de estos factores de riesgo en una misma persona, coinciden con lo hallado en otros estudios (30). Es especialmente relevante si se toma en consideración que la población en estudio está conformada por adultos jóvenes, lo cual justifica plenamente la necesidad de realizar intervenciones específicas en este grupo de población con programas de promoción en salud, a fin de lograr la disminución de dichos factores de riesgo, conclusión similar a la que arribaron otros investigadores $(31,32)$.

En conclusión, las condiciones socioeconómicas específicas del grupo estudiado permiten que estos resultados se extrapolen, aunque dentro de ciertos límites especialmente debidos al tamaño de la muestra y al elevado porcentaje de mujeres incluidas, a una parte importante de la población de adultos jóvenes de Cartagena de Indias, que muy seguramente comparten los perfiles demostrados en este estudio y coinciden con lo hallado en estudios de gran alcance $(33,34)$. En tal sentido, se hace relevante tomarlos en consideración en la medida que evidencian, dentro del grupo etario de adultos jóvenes, comportamientos críticos en lo que respecta a la presencia de factores de riesgo que contribuyen directa o indirectamente a sostener la progresión nefasta que han mostrado estas enfermedades en la ciudad y en el mundo, en los últimos años.

Por otra parte, justifican plenamente la necesidad y permiten prever el elevado impacto que pudieran tener las campañas y estrategias de salud que tengan como objetivo el control y la reducción del conjunto de factores de riesgo en edades tempranas, si se considera que resulta más fácil inculcar hábitos saludables en los jóvenes que modificar comportamientos en la edad adulta o luego del inicio de la enfermedad (35).

En ese orden de ideas, estos resultados se convierten en una línea de base para el estudio de enfermedades cardiovasculares, que puede ser aprovechada por la comunidad académica, científica y por los estamentos públicos y privados para derivar macroproyectos que beneficien a la población, no sólo en el sentido de prevención de riesgos, sino en abordajes comprensivos de las condiciones socioculturales y de accesibilidad en salud para esta población que pudieran, en el futuro, hacerse extensibles a grupos de población con características y comportamientos similares a los del estudiado. 


\section{Conflictos de interés}

Los autores manifiestan que no existen conflictos de intereses en la elaboración y ejecución de este proyecto.

\section{Financiación}

Este proyecto fue financiado en su totalidad con recursos propios de los investigadores.

\section{Referencias}

1. Molina D. Propuesta en prevención del riesgo cardiovascular. Revista Colombiana de Cardiología. 2008;15:203-6.

2. Organización Panamericana de la Salud. La salud en las Américas. Volumen I. (Publicación Científica No. 569). Washington, D.C.: OPS; 1998. p. 168-75. Fecha de consulta: 26 de junio de 2007. Disponible en: http://www. paho.org/spanish/HIA1998/SaludVol1.pdf.

3. Duran Á, Martínez M, Rodríguez D, Mosquera W, VillaRoel C. Estudio del riesgo cardiovascular en la infancia a través de un modelo clínico-investigativo. Revista Colombiana de Cardiología. 2006;13:128-31.

4. Villavicencio C. Trends in preventative interventions: Promoting heart health in clinical practice. Carmen Program, Región del Bío Bío, Chile. Chile: Secretaría Regional Ministerial de Salud, Gobierno de Chile; 2004. Fecha de consulta: 25 de marzo de 2009. Disponible en: http://www. nhlbi.nih.gov/health/prof/heart/other/paho/carmen_chile.pdf

5. Organización Panamericana de la Salud. CARMEN: conjunto de acciones para la reducción y el manejo de las enfermedades no transmisibles. Fecha de consulta: 27 de marzo de 2009. Disponible en: http://www.paho.org/spanish/ $\mathrm{ad} / \mathrm{dpc} / \mathrm{nc} /$ carmen-info.htm

6. Organización Panamericana de la Salud, Organización Mundial de la Salud. Promoción de la salud cardiovascular en las Américas. Enfermedades crónicas: prevención y control en las Américas. 2007;1:4-6. Fecha de consulta: 8 de agosto de 2008. Disponible en: http://www.paho.org/ spanish/AD/DPC/NC/cronic-2007-1-1.pdf.

7. Texas Heart Institute. Factores de riesgo cardiovascular. Fecha de consulta: 27 de marzo de 2007. Disponible en: http://www.texasheartinstitute.org/HIC/Topics_Esp/HSmart/ riskspan.cfm.

8. Lee D, Chiu M, Manuel D, Tu K, Wang X, Austin P, et al. Trends in risk factors for cardiovascular disease in Canada: temporal, socio-demographic and geographic factors for the Canadian Cardiovascular Outcomes Research Team. CMAJ. 2009;181:E55-66.

9. Sánchez-Contreras M, Moreno-Gómez G, Marín-Grisales M, García-Ortiz L. Factores de riesgo cardiovascular en poblaciones jóvenes. Rev Salud Pública. 2009;11:110-22.

10. Valdez R, Greenlud KJ, Khoury MJ, Yoon PW. Is family history a useful tool for detecting children at risk for diabetes and cardiovascular diseases? A public health perspective. Pediatrics. 2007;120:S78-86.

11. California Pacific Medical Center. Insuficiencia cardiaca congestiva. California Pacific Medical Center, 2005. Fecha de consulta: 10 de junio de 2009. Disponible en: http://www. cpmc.org/learning/documents/rg-chf-span.pdf. 12.
Michos E, Nasir K, Rumberger J, Vasamreddy C, Braunstein J, Budoff M, et al. Relation of family history of premature coronary heart disease and metabolic risk factors to risk of coronary arterial calcium in asymptomatic subjects. Am J Cardiol. 2005;95:655-7.

13. Zimmet $\mathbf{P}$, Alberti G, Serrano $\mathbf{M}$. Una nueva definición mundial del síndrome metabólico propuesta por la Federación Internacional de Diabetes: fundamento y resultados. Rev Esp Cardiol. 2005;58:1371-6.

14. National Institute of Health. National Cholesterol Education Program. Third Report of the Expert Panel on Detection, Evaluation and Treatment of High Blood Cholesterol in Adults. (Adult Treatment Panel III) ATP III Guidelines AtA-Glance Quik Desk Reference. Fecha de consulta: 10 de diciembre de 2009. Disponible en: http://www.nhlbi.nih.gov/ guidelines/cholesterol/atglance.pdf.

15. Ardila E, Hernández E. Consenso Colombiano de Síndrome Metabólico. Bogotá D.C.: Asociación Colombiana de Endocrinología; 2006.

16. Secretaría de Salud/Subsecretaría de Prevención y Protección de la Salud. Programa de acción: enfermedades cardiovasculares e hipertensión arterial. $1^{\underline{a}}$ edición. México D.F: Secretaría de Salud; 2001. Fecha de consulta: 26 de julio de 2007. Disponible en: http://bibliotecas.salud.gob. mx/gsdl/collect/publin1/index/assoc/HASH0155.dir/doc.pdf.

17. Girotto C, Vacchino M, Spillmann C, Soria J. Prevalencia de factores de riesgo cardiovascular en ingresantes universitarios. Rev Saude Pública. 1996;30:576-86.

18. Martínez-Palomino G, Vallejo M, Huesca C, Álvarez E, Paredes G, Lerma C. Factores de riesgo cardiovascular en una muestra de mujeres jóvenes mexicanas. Arch Cardiol Méx. 2006;76:401-7.

19. Bustos P, Amigo H, Arteaga A, Acosta A, Rona R. Factores de riesgo de enfermedad cardiovascular en adultos jóvenes. Rev Med Chile. 2003;131:973-80.

20. Mora G. Dieta y enfermedad coronaria. Revista de la Facultad de Medicina de la Universidad Nacional de Colombia. 2005;53:98-116.

21. Neumark-Sztainer D, Wall M, Perry C, Story M. Correlates of fruit and vegetable intake among adolescents. Findings from Project EAT Prev Med. 2003;37:198-208.

22. Bensimhon DR, Kraus WE, Donahue MP. Obesity and physical activity: A review. Am Heart J. 2006;151:598-603.

23. Fajardo H, Gutiérrez A, Navarrete S, Barrera J. Prevalencia factores de riesgo cardiovascular. Localidad de los Mártires. Bogotá-Colombia. Revista de la Facultad de Medicina de la Universidad Nacional de Colombia. 2003;51:198-202.

24. González GR, Alcalá RJ. Consumo de alcohol y salud pública. Rev Fac Med UNAM. 2006;49:238-41.

25. Klatsky A, Udaltsova N. Alcohol drinking and total mortality risk. Ann Epidemiol. 2007;17:S63-7.

26. Rimm E, Moats C. Alcohol and coronary heart disease: Drinking patterns and mediators of effect. Ann Epidemiol. 2007;17:S3-7.

27. Vera JJ. Enfermedad vascular cerebral isquémica en adultos jóvenes: etiología y factores de riesgo. Hospital Nacional Guillermo Almenara Irigoyen, 1998-2001 (tesis). Lima: Universidad Nacional Mayor de San Marcos; 2002. p. 
54. Fecha de consulta: 1 de diciembre de 2008. Disponible en: http://sisbib.unmsm.edu.pe/bibvirtualdata/Tesis/Salud/ Vera_RJ/enPDF/T_completo.pdf.

28. Soto V, Vergara E, Neciosup E. Prevalencia y factores de riesgo de síndrome metabólico en población adulta del departamento de Lambayeque, Perú - 2004. Rev Per Med Exp Sal Pub. 2005;22:254-61.

29. Asociación Latinoamericana de Diabetes. Guías ALAD de diagnóstico, control y tratamiento de la diabetes mellitus tipo 2. ALAD, 2007. Fecha de consulta: 3 de agosto de 2008. Disponible en: http://www.fenadiabetes.org.ve/docs/ guia.pdf.

30. Pereira J, Barreto S, Passos V. Perfil de risco cardiovascular e autoavaliação da saúde no Brasil: estudo de base populacional. Rev Panam Salud Publica. 2009;25:491-8.

31. Sirit Y, Acero C, Bellorin M, Portillo R. Síndrome metabólico y otros factores de riesgo cardiovascular en trabajadores de una planta de policloruro de vinilo. Rev Salud Pública. 2008;10:239-49.
32. Díaz-Realpe JE, Muñoz-Martínez J, Sierra-Torres $\mathbf{C H}$. Factores de riesgo para enfermedad cardiovascular en trabajadores de una institución prestadora de servicios de salud, Colombia. Rev Salud Pública. 2007:9:64-75.

33. Lanas F, Avezum A, Bautista L, Diaz R, Luna M, Islam $\mathbf{S}$, et al. INTERHEART Investigators in Latin America. Risk factors for acute myocardial infarction in Latin America: The INTERHEART Latin American Study. Circulation. 2007;115:1067-74.

34. Karlamangla A, Singer B, William D, Schwartz J, Matthew K, Kiefe I, et al. Impact of socioeconomic status on longitudinal accumulation of cardiovascular risk in young adults: the CARDIA Study (USA). Soc Sci Med. 2005;60:9991015.

35. Sánchez-Contreras M, Moreno-Gómez G, Marín-Grisales M, García-Ortiz L. Factores de riesgo cardiovascular en poblaciones jóvenes. Rev Salud Pública. 2009;11:110-22. 